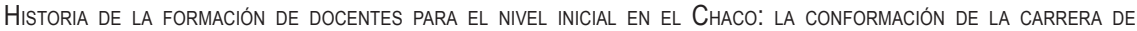
Profesorado de Educación Pre-elemental en la Universidad Nacional del Nordeste (1973-1982). Páginas 65-78 en Revista de la Escuela de Ciencias de la Educación, año12, nRo.11, vol.1, enero a Junio de 2016. ISSN 18516297. ISSN EN LINEA 2362-3349.

\title{
HISTORIA DE LA FORMACIÓN DE DOCENTES PARA EL NIVEL INICIAL EN EL CHACO: LA CONFORMACIÓN DE LA CARRERA DE PROFESORADO DE EDUCACIÓN PRE-ELEMENTAL EN LA UNIVERSIDAD NACIONAL DEL NORDESTE (1973-1982)(1)
}

\author{
Alcides David Musín* \\ Universidad Nacional del Nordeste, Argentina. \\ alcidesmusin@gmail.com \\ lleana Ramírez** \\ Universidad Nacional del Nordeste, Argentina. \\ ileana_rz@hotmail.com \\ Victoria Soledad Almiron*** \\ Universidad Nacional del Nordeste, Argentina. \\ sol_almiron@live.com.ar
} Recibido: 10/03/2016 Aceptado: 15/04/2016

* Profesor en Ciencias de la Educación (Universidad Nacional del Nordeste - UNNE); Especialista y Diplomado Superior en Ciencias Sociales con mención en Currículum y Prácticas Escolares en Contexto (FLACSO Argentina); Maestrando en Ciencias Sociales (Universidad Nacional del Litoral). Profesor Adjunto Ordinaria de la asignatura "Historia de la Educación Infantil" del profesorado y licenciatura en Educación Inicial (Departamento de Educación Inicial - Facultad de Humanidades de la UNNE).

** Profesora en Ciencias de la Educación y Especialista en Docencia Universitaria por la Universidad Nacional del Nordeste. Doctoranda en la Carrera Doctorado en Educación en la Facultad de Humanidades de la Universidad Nacional de Entre Ríos. Profesora Auxiliar Docente de Primera Categoría en la cátedra Historia de la Educación Infantil en las carreras Profesorado y Licenciatura en Educación Inicial del Departamento de Educación Inicial de la Facultad de Humanidades de la Universidad Nacional del Nordeste.

*** Profesora en Ciencias de la Educación; Especialista y Diplomada Superior en Ciencias Sociales con mención en Lectura, Escritura y Educación, FLACSO-Argentina; Doctoranda en Ciencias Sociales por la Universidad Nacional de Entre Ríos. Realiza actividades de docencia en el nivel superior como auxiliar docente en la cátedra "Historia de la Educación Infantil" de la Facultad de Humanidades, Universidad Nacional del Nordeste (Chaco, Argentina). 
Revista de la Escuela de Ciencias de la Educación, año12, nRo.11, vol. 1, enero a Junio de 2016. Páginas 65-78. ISSN 1851-6297. ISSN EN LINEA 2362-3349. HISTORIA DE LA FORMACIÓN DE DOCENTES PARA EL NIVEL INICIAL EN EL CHACO: la conformación de la carrera de Profesorado de Educación Pre-elemental en la Universidad Nacional del Nordeste (1973-1982). Alcides David Musín - Ileana Ramirez - Victoria Soledad Almiron

\title{
Resumen
}

La formación de docentes para el nivel inicial en el Chaco reconoce un recorrido que incluye preocupaciones provinciales y nacionales, plasmadas en propuestas formativas en las décadas de los ' 60 y ' 70 que dan cuenta de perspectivas teóricas diferenciadas que, aparentemente, han convivido de manera "armónica" en la definición de lo que hoy conocemos como Profesorado de Educación Inicial.

Nos interesa indagar acerca de ¿qué características adquirió la formación docente para el nivel pre-elemental en el contexto chaqueño de los '70?, ¿qué tensiones existieron en la construcción identitaria de la formación de docentes para el nivel en el Chaco? A partir de ello describimos los actores e instituciones intervinientes, los sentidos y significados dados a la educación infantil en sus orígenes, el rol docente, entre otros, para aportar a la construcción de conocimientos historiográficos desde una perspectiva regional. Principalmente, la trayectoria de formación y accionar de Nélida Sosio de Iturrrioz nos resultaron relevantes para comprender los sentidos fundacionales en la creación de la carrera de formación docente para el nivel.

Recurrimos a diversas fuentes primarias tales como entrevistas semi estructuradas a docentes y ex alumnas de la carrera, documentos institucionales e informes de época sobre el nivel en el Chaco.

\section{Palabras clave:}

Formación docente - Sentidos fundacionales - Educación inicial - Universidad - Chaco.

\begin{abstract}
Teacher training for the initial level in Chaco recognizes a pathway that includes provincial and national concerns, reflected in training proposals in the decades of the 60 s and 70 s that account for different theoretical perspectives that have apparently lived so harmonica in the definition of what we now know as Initial Teacher Education.

We are interested in inquiring about what characteristics acquired the teacher training for pre-elementary level in the context of the 70s in Chaco?, what tensions existed in the identity construction of teacher training for the level in this province? From this view we describe the actors and institutions involved, senses and meanings given to children's education in its origins, the role of teachers, among others, to contribute to the construction of historiographical knowledge from a regional perspective. Mainly, the trajectory of formation and actions of Nelida Sosio de Iturrioz were relevant to understanding the foundational ways in creating the teacher training career for the initial level.

We use various primary sources such as semi-structured interviews to teachers and alumnae of the career, institutional documents and epoch reports on the initial level in Chaco.
\end{abstract}

\section{Key words:}

Teacher training - Foundational senses - Initial education - University - Chaco. 
Revista de la Escuela de Ciencias de la Educación, año 12, nRo.11, vol. 1, enero a junio de 2016. Páginas 65-78. ISSN 1851-6297. ISSN EN LINEA 2362-3349. HISTORIA DE LA FORMACIÓN DE DOCENTES PARA EL NIVEL INICIAL EN EL CHACO: la conformación de la carrera de Profesorado de Educación Pre-elemental en la Universidad Nacional del Nordeste (1973-1982). Alcides David Musín - Ileana Ramirez - Victoria Soledad Almiron

\section{Un recorrido por la trayectoria de formación de Nélida Sosio de Iturrioz:} la impronta de su formación

En los orígenes del profesorado de Educación Pre-elemental de la Universidad Nacional del Nordeste (1973) (2), la Prof. Nélida Sosio de Iturrioz ocupó un lugar central como impulsora, realizando acciones de elaboración del plan curricular, organización y posterior desarrollo de la carrera. Su accionar se constituyó en un factor importante para instalar a nivel social y académico la necesidad de generar instancias de formación docente específica para el nivel.

Oriunda de Buenos Aires y formada como Profesora Nacional de Jardines de Infantes en el Instituto "Sara Ch. de Eccleston", se radicó en el Chaco a fines de los '60 y comenzó a trabajar en el jardín de infantes provincial $N^{\circ} 5$. En el año 1972, en calidad de Secretaria Técnica del Departamento de Educación Pre-elemental (3) de la Facultad de Humanidades, elaboró un informe (4) donde expuso sobre el estado de la educación pre-elemental en la provincia, señalando, entre otras cuestiones, la necesidad de expandir los jardines de infantes y de contar con personal especializado dado que hasta ese entonces quienes trabajaban en las salas de jardines eran Maestras Normales Nacionales. En virtud de ello, y en sintonía con las recomendaciones del Primer Congreso Argentino de la Organización Mundial de la Educación Pre Escolar en 1968 (5), se recomienda la creación del "Profesorado de Educación Pre-elemental" en el ámbito de la Facultad de Humanidades de la Universidad Nacional del Nordeste (en adelante UNNE).

A partir de lo expuesto, nos interesa describir algunos rasgos centrales de la formación docente de Nélida, ya que entendemos que la misma ha tenido una influencia destacada en la formulación de una propuesta de formación específica para el nivel en el Chaco (y la región del noreste argentino), y en las características pedagógico-curriculares que fue adquiriendo el profesorado en la etapa de constitución y posterior desarrollo.

Nélida ingresa al Instituto Superior de Profesorado "Sara Ch. de Eccleston" en 1954. En 1955 se recibe de Profesora Normal Nacional de Jardines de Infantes.

En su relato retrospectivo, Nélida refirió sobre las modalidades de ingreso a la carrera, la serie de exámenes que tenían que pasar y los conocimientos elementales que eran solicitados:

El lunes me fui al profesorado con guardapolvo blanco, eso sí, había que ir con guardapolvo blanco, como maestra (...) nos tomaron el primer día el examen de letras, de literatura, con una composición que teníamos que escribir sobre el tema: 'si no fuera quien soy, quién y cómo me gustaría ser'. El segundo día era de psicología (...) y al día siguiente tuvimos examen de música y nos hicieron solfear y cantar (...) otro día fue de dibujo (...) examen de francés (...) 
Revista de la Escuela de Ciencias de la Educación, año12, nRo.11, vol. 1, enero a Junio de 2016. Páginas 65-78. ISSN 1851-6297. ISSN EN LINEA 2362-3349. HISTORIA DE LA FORMACIÓN DE DOCENTES PARA EL NIVEL INICIAL EN EL CHACO: la conformación de la carrera de Profesorado de Educación Pre-elemental en la Universidad Nacional del Nordeste (1973-1982). Alcides David Musín - Ileana Ramirez - Victoria Soledad Almiron

Te daban un grupo de chicos, delante de ese tribunal, que te helaba (...) Nos hacían contar un cuento delante de un grupo de 10 chiquitos, sin láminas, contando (entrevista a Nélida Sosio, 2013).

Saberes relacionados con lengua, literatura, psicología, música, dibujo e idioma extranjero eran parte de los conocimientos solicitados para el ingreso. Esta instancia también buscaba vislumbrar saberes vinculados con la práctica, como el trabajo grupal con niños que estarían presentes en su futuro ejercicio profesional.

Describe una formación compleja, disciplinada, y donde la figura de Margarita Ravioli era central:

El profesorado era muy, muy estricto. Íbamos a la mañana, nos recogía un ómnibus en plaza Italia y ella era el alma mater del profesorado pero no era una mujer estrella, ¿cómo te podría decir? (...) tenía mucha personalidad (...) Margarita era profesora de Didáctica nuestra, entonces nos controlaba las prácticas, nos organizaba que íbamos a hacer, nos estudiaba minuciosamente a cada una. En las vacaciones, tanto de invierno como de verano, nos daba a cada una un libro diferente pero de la biblioteca de ella, para que lo leyéramos y lo trabajáramos, lo estudiáramos (Ibídem).

Como también se puede observar en la cita, la práctica de lectura aparece como un eje estructurante para la formación; la creencia de que la misma y la formación cultural eran pilares imprescindibles en la formación de las futuras docentes de nivel pre-elemental:

Nos daba libros tradicionales sobre Pestalozzi, Fröebel, nos hacía leer mucho Pestalozzi, nos hacía leer muchas historias sobre María Montessori, sobre el material de María Montessori, las hermanas Agazzi (...), hacía hincapié en que leyéramos mucho y nos daba romances españoles anónimos y nos hacía memorizarlos, porque nos decía que eso nos enriquecía espiritualmente y nos enriquecía la imaginación y que en el jardín, la maestra jardinera tiene que tener imaginación, porque si no tiene imaginación no servía para estar con los chicos. Entonces ella decía que era importante organizarse cuando conocías el grupo y organizarse en el sentido de qué le voy a dar a este grupo, pero que los temas que eligiera para darle a esos chicos tenían que estar de acuerdo a su realidad (...) (Ibídem).

Tanto la práctica de la lectura para la formación como las lecturas promocionadas desde estos espacios, nos acercan a los sentidos y significados dados a la identidad profesional y al quehacer docente del nivel. La profesora Ravioli recuperaba la fecundidad de concepciones sobre la infancia y la educación infantil de los precursores universales, Juan Jacobo Rousseau, Juan 
Revista de la Escuela de Ciencias de la Educación, año 12, nRo.11, vol. 1, enero a junio de 2016. Páginas 65-78. ISSN 1851-6297. ISSN EN LINEA 2362-3349. HISTORIA DE LA FORMACIÓN DE DOCENTES PARA EL NIVEL INICIAL EN EL CHACO: la conformación de la carrera de Profesorado de Educación Pre-elemental en la Universidad Nacional del Nordeste (1973-1982). Alcides David Musín - Ileana Ramirez - Victoria Soledad Almiron

Enrique Pestalozzi, Federico Fröebel, María Montessori, las hermanas Rosa y Carolina Agazzi, Ovidio Decroly. Avanzó hacia la construcción de nuevas fronteras fundacionales referidas, por ejemplo, a la formación docente vinculada con el Jardín Maternal y a la articulación de la sala de 5 años con el primero y el segundo grado de la Educación Primaria. Desde esta perspectiva de formación para el nivel, el reconocimiento del grupo y la búsqueda de vinculación con la realidad circundante del niño/la niña serían tareas necesarias para la organización escolar en el nivel.

Asimismo, entendemos que el relato de Nélida evidencia el lugar preponderante dado a la formación pedagógica más academicista, pero que a la vez rompe con una concepción de "academicismo cerrado" al incorporar la práctica en un nivel de igualdad a la formación en contenidos:

Por ejemplo el 'Emilio' de Rousseau, todas esas tienen ideas muy buenas, ella sí nos decía que Rousseau era muy teórico, pero en la práctica no había sido tan efectivo como en su teoría. Lo mismo nos decía de Pestalozzi, ella nos decía que Pestalozzi tenía ideas educativas muy buenas pero no había sido apoyado por la sociedad pero tampoco Fröebel. Entonces nos decía que cuando llegamos a una sociedad y queremos imponer algo hay que ser coherente con lo que se impone y las ideas siempre tenían que estar acompañadas por la acción (...).

Es que llegabas como alumna y ella ya te metía en una sección a practicar, a ayudar a limpiar la cola a los chicos, a darle de comer en el comedor, a vivir ahí, entonces vivíamos en el profesorado (Ibídem).

Como lo habíamos anticipado, ya en el examen para el ingreso al Instituto se solicitaban actividades de trabajo con niños/niñas, es decir, la presencia de la práctica como un eje constante y estructurante.

Otro rasgo de la formación que aparece como relevante es el carácter fuertemente filosófico que presentaba la misma: por ejemplo en el examen de ingreso debían escribir un ensayo cuyo tema era: "si no fuera quien soy, ¿quién y cómo me gustaría ser?”. Una invitación a pensarse y a proyectarse ¿qué habrán escrito esas jóvenes de los cincuenta en Argentina?

Estos rasgos centrales de la formación recuperados desde el relato de Nélida nos sugieren su imbricación con un debate fundante en la formación de docentes para el nivel inicial. En este sentido, compartimos con Diker y Terigi (2003) que la excelencia en la formación tiene que ir abriéndose lugar dentro de la polémica acerca del valor educativo del nivel inicial.

En la historia educativa argentina hubo una marcada centralidad de la educación primaria en el desarrollo de nuestro sistema educativo nacional. Ello explicaría el énfasis puesto en la formación de docentes para este nivel a través de las Escuelas Normales, frente a un proceso de institucionalización 
Revista de la Escuela de Ciencias de la Educación, año 12, nRo.11, vol. 1, enero a junio de 2016. Páginas 65-78. ISSN 1851-6297. ISSN EN LINEA 2362-3349. HISTORIA DE LA FORMACIÓN DE DOCENTES PARA EL NIVEL INICIAL EN EL CHACO: la conformación de la carrera de Profesorado de Educación Pre-elemental en la Universidad Nacional del Nordeste (1973-1982). Alcides David Musín - Ileana Ramirez - Victoria Soledad Almiron

mucho menos sistemático de la formación de docentes para otros niveles. La historia del proceso de institucionalización de la formación docente específica para el nivel inicial está atravesado por un histórico dilema que afecta a la identidad del nivel en cuanto tal, y que puede resumirse en la discusión entre el carácter educativo o doméstico del jardín de infantes, surcada por fuertes cuestionamientos provenientes del normalismo positivista hacia los postulados espiritualistas que promovían los jardines, lo que trajo aparejado el cierre de los profesorados y el decrecimiento cuantitativo de los jardines de infantes a finales del siglo XIX (Carli, 2002; Diker y Terigi, 2003; Ponce, 2006). A su vez, esta polémica sobre la función del nivel repercutió en debates acerca del peso que corresponde, en los planes de estudio, a la formación llamada 'pedagógica' (centrada en la función educativa del jardín) frente a una capacitación de tipo puramente 'asistencial' (centrada en el entretenimiento y el cuidado de los niños).

Hacia comienzos del siglo XX, en parte debido a las mencionadas críticas efectuadas al Jardín de Infantes, el Estado no había avanzado en definiciones políticas (institucionales y pedagógicas) para el nivel inicial. Recién a partir de la década del ' 30 comienza a percibirse en el país un cambio paulatino en las políticas educativas en relación a este nivel, puntualmente a partir de algunos ensayos y experiencias en torno a propuestas escolanovistas europeas (por caso la pedagogía montessoriana y la pedagogía decroliana) y, es en este contexto, donde tiene lugar la creación del Profesorado "Sara Ch. de Eccleston" y el Jardín de Infancia "Mitre". El Instituto Eccleston ha sido pionero en la formación docente para el nivel inicial. Comienza su actividad el 8 de abril de 1937 en respuesta a los reclamos que realizara la "Asociación Pro-difusora del Kindergarten" por la ausencia de instituciones de formación docente para el nivel (6) (Diker y Terigi, 2003; Vasta y Gispert, 2009).

La formación de Nélida transcurre durante el gobierno peronista (19461955). Este período significó un avance considerable en políticas para la infancia. Destacamos de ello tres puntos: el reconocimiento de la infancia, vinculado con la proyección de un nuevo sujeto político; la expansión cuantitativa del nivel inicial (7); y como tercer y último punto, la sanción de la Ley Simini N 5096/46 para la provincia de Buenos Aires, donde por primera vez aparece expresado como política desde el plano legislativo la formación docente para el nivel, la obligatoriedad y gratuidad escolar de las salas de 3, 4 y 5, y el reconocimiento del docente como trabajador de la educación.

Es en este contexto y espacio institucional donde Nélida Sosio de Iturrioz inicia su trayectoria de formación específica para el nivel y también ejerció sus primeros años de docencia, ya que trabajó durante cuatro años en el Jardín de Infancia "Mitre". 
Revista de la Escuela de Ciencias de la Educación, año 12, nRo.11, vol. 1, enero a junio de 2016. Páginas 65-78. ISSN 1851-6297. ISSN EN LINEA 2362-3349. HISTORIA DE LA FORMACIÓN DE DOCENTES PARA EL NIVEL INICIAL EN EL CHACO: la conformación de la carrera de Profesorado de Educación Pre-elemental en la Universidad Nacional del Nordeste (1973-1982). Alcides David Musín - Ileana Ramirez - Victoria Soledad Almiron

Desde Buenos Aires al Chaco. La influencia de Margarita Ravioli en los sentidos fundacionales del Profesorado de Educación Pre-elemental

En el año 1968 Nélida se traslada con su familia para residir en el Chaco con un mandato muy fuerte de quien fuera su formadora: "y Margarita me dijo: vayan, vayan y vos tenés que crear el profesorado allá", "Continuamente mantuve contacto con Margarita, yo viajaba mucho a Buenos Aires porque tenía toda mi familia y cada vez que iba charlaba con ella y ella me insistía", refiriéndose a la creación de un profesorado.

Como punto de partida consideramos necesario introducir una primera descripción del estado de situación de la educación inicial en la provincia (8), que aportará a la comprensión y análisis de los sentidos fundacionales del Profesorado de Educación Pre-elemental en la UNNE, desde una perspectiva que incluye las características socio-educativas provinciales.

En el año 1954 se crea el Jardín de Infantes provincial №1 "Merceditas de San Martín (9)", una institución autónoma independiente de escuela primaria alguna. Allí fueron designadas como maestras organizadoras las Profesoras en Jardines de Infantes Liam C. de Leonhard y Berta Bocco de Varisco.

En el Jardín de Infantes $N^{\circ} 1$ funcionaba el "Centro de Actualización en Educación Pre-elemental", que en el año 1961 dicta el primer curso de actualización docente para el personal del establecimiento basado en los principios de la escuela nueva. El mismo estuvo a cargo de la Inspectora Técnica de la provincia de Tucumán, Profesora Soledad María Ardiles Gray de Stein. A partir de esta acción se dictan nuevos cursos de perfeccionamiento de forma periódica, para satisfacer las necesidades del momento, tanto en Resistencia como en el interior de la provincia, con la siguiente carga horaria: en 1963, con 159 horas de clase; en 1965-66, con 300 horas; y en 1968, con 178 hs.

Hacia 1967 se crea en la provincia la Inspección Técnica - Área Preelemental, dependiente del Consejo General de Educación. En este período la educación pre-escolar se fue expandiendo, atendiéndose a infantes de 3 a 5 años en distintos establecimientos dependientes de la Nación, la Provincia o instituciones privadas. La Nación organizaba, controlaba y sostenía las secciones pre-escolares anexas a las escuelas primarias, por intermedio del Consejo Nacional de Educación, y las pertenecientes a los establecimientos que funcionaban en Escuelas Normales, por intermedio de la Subsecretaría de Educación de la Nación. La Provincia, a través del Consejo General de Educación, sostenía dos tipos de establecimientos del nivel: a). Anexos a grupos escolares, bibliotecas y a Escuelas Normales; b). Autónomos.

Por su parte, algunas instituciones privadas contaban en su organización con Secciones de nivel pre-escolar, no todas sujetas al contralor del Estado nacional o provincial, pero sí subvencionadas regularmente por los organismos oficiales. 
Revista de la Escuela de Ciencias de la Educación, año12, nRo.11, vol. 1, enero a Junio de 2016. Páginas 65-78. ISSN 1851-6297. ISSN EN LINEA 2362-3349. HISTORIA DE LA FORMACIÓN DE DOCENTES PARA EL NIVEL INICIAL EN EL CHACO: la conformación de la carrera de Profesorado de Educación Pre-elemental en la Universidad Nacional del Nordeste (1973-1982). Alcides David Musín - Ileana Ramirez - Victoria Soledad Almiron

Según lo señalaba la entrevistada, la provincia del Chaco fue la primera jurisdicción en crear jardines de infantes autónomos en el país.

El cuadro que presentamos a continuación refiere a la cantidad de jardines existentes en la provincia en los años 1968 y 1971, distribuidos según modalidad de gestión y ámbito de dependencia institucional:

\begin{tabular}{|l|l|}
\hline & \\
Año 1968 & Año 1971 \\
\hline
\end{tabular}

\begin{tabular}{|l|c|c|l|c|c|}
\hline & $\begin{array}{c}\text { Jardines } \\
\text { autónomos }\end{array}$ & $\begin{array}{c}\text { Jardines } \\
\text { anexos }\end{array}$ & $\begin{array}{c}\text { Jardines } \\
\text { autónomos }\end{array}$ & $\begin{array}{c}\text { Jardines } \\
\text { anexos }\end{array}$ \\
\hline Nacional & - & 6 & Nacional & - & 10 \\
\hline Provincial & 5 & 11 & Provincial & 10 & 11 \\
\hline Privada & 4 & 16 & Privada & 4 & 18 \\
\hline Total & 9 & 33 & Total & 14 & 39 \\
\hline
\end{tabular}

(Informe, 1972: 17)

Según se observa en los datos, entre los años 1968 y 1971 hubo una ampliación de la educación pre-escolar en la provincia del Chaco, que pasó de 42 a 53 establecimientos educativos del nivel (10). Respecto a la modalidad de gestión, había una cantidad similar de jardines estatales y privados; la intervención del Estado Nacional sólo se dio en la creación y sostenimiento de jardines anexos.

Retomamos del informe otros datos de contexto que a continuación se detallan. En cuanto a las docentes que se desempeñaban en estas instituciones, vale señalar que para el año 1972 un $80 \%$ eran Maestras Normales con certificado de curso de capacitación y sólo el $20 \%$ contaba con un profesorado especializado para la educación pre-escolar.

La matrícula escolar para el año 1968 era de 1.904 y en 1971, de 2.203 estudiantes. Esta matrícula representaba aproximadamente el $6.30 \%$ de la población total de niños/niñas en la provincia.

Referido a los factores socio-económicos, como la colaboración de los/ las niños/niñas en las tareas familiares, su utilización como fuerza de trabajo (cosecheros, lustrabotas, mendigos, etc.) o el traslado frecuente de las familias para encontrar mejoras económicas (conocidos como los pobladores "golondrinas"), entre otros, incidieron en la baja incorporación de esta población en la educación pre-escolar. Asimismo, otro factor que podría contribuir a dicho fenómeno es que hacia el año 1972 la provincia todavía carecía de una ley de educación provincial y estaba en vigencia la Ley 1420/84, la cual no fijaba la obligatoriedad escolar del jardín de infantes. 
Revista de la Escuela de Ciencias de la Educación, año 12, nRo.11, vol. 1, enero a junio de 2016. Páginas 65-78. ISSN 1851-6297. ISSN EN LINEA 2362-3349. HISTORIA DE LA FORMACIÓN DE DOCENTES PARA EL NIVEL INICIAL EN EL CHACO: la conformación de la carrera de Profesorado de Educación Pre-elemental en la Universidad Nacional del Nordeste (1973-1982). Alcides David Musín - Ileana Ramirez - Victoria Soledad Almiron

\section{Nélida y la educación pre-escolar en el Chaco}

Inmediatamente a su llegada a la provincia, Nélida comienza a trabajar como docente en uno de los 5 jardines de infantes autónomos que estaban funcionando. Sin embargo, como lo expresa en su relato, siempre tuvo una marcada preocupación por organizar un profesorado para la formación específica para la educación pre-escolar:

(...) la cuestión es que empecé a jorobar, como yo ya tenía la titularidad me relevaron de las tareas y me mandaron a la Facultad de Humanidades a trabajar con el profesor Schmid como Secretaria Técnica del Departamento de Educación Pre- elemental para organizar el Profesorado. Entonces ahí me aboque... estos señores de la Facultad de Humanidades me pidieron que lo fundamente porque sin fundamento no podía. Entonces empecé a trabajar con estadísticas, las cuatro provincias, es decir de Misiones, Chaco, Corrientes y Formosa y el norte de Santa Fe (entrevista a Nélida Sosio, 2013).

En esta línea, la primera acción que realiza, y que es antecedente directo de lo que posteriormente será el profesorado en Educación Pre-elemental, fue el dictado de un curso de capacitación para el nivel que duró seis meses con una carga horaria de 300 horas. Ella comenta: "(...) se anotaron un montón, lo hizo un curso muy lindo y mientras tanto yo pensaba bueno, yo tengo que crear el profesorado, y Margarita me pinchaba (...)". El nuevo curso se dictó a partir del 3 de agosto en 1970, con una cantidad de 65 alumnas regulares de las cuales 55 terminaron en el mes noviembre de ese mismo año.

Las profesoras dictantes eran de la Facultad de Humanidades, y otras provenían de Buenos Aires, del Instituto "Sara Ch. de Eccleston", y de Tucumán, representada por Soledad María Ardiles Gray de Stein (Mery Stein), quien seguía la tradición escolanovista norteamericana. Al respecto menciona Nélida una primera línea de disputa en la génesis y constitución de este curso:

Yo organizo un curso pero yo traigo profesores de Buenos Aires pero no... No acá para capacitar hay que capacitar bien, hay que traer gente de Buenos Aires... porque Margarita Ravioli impulsó la Educación Nueva desde Buenos Aires. Pero en Tucumán estaba Mery Stein (...) porque Berta era de la camada de la señora de Stein en Tucumán, entonces quería que estuviera esa línea, entonces yo dije: 'vos pones esto y yo pongo esto' (Ibídem).

En las decisiones respecto a la organización de ese curso ya se ponen de manifiesto las distintas tradiciones pedagógicas, didácticas y filosóficas sobre el jardín de infantes.

Posteriormente la Facultad de Humanidades puso a consideración la estructuración de las carreras para el período 1971-1975, entre las cuales figuraba 
Revista de la Escuela de Ciencias de la Educación, año 12, nRo.11, vol. 1, enero a junio de 2016. Páginas 65-78. ISSN 1851-6297. ISSN EN LINEA 2362-3349. HISTORIA DE LA FORMACIÓN DE DOCENTES PARA EL NIVEL INICIAL EN EL CHACO: la conformación de la carrera de Profesorado de Educación Pre-elemental en la Universidad Nacional del Nordeste (1973-1982). Alcides David Musín - Ileana Ramirez - Victoria Soledad Almiron

como prioritaria el curso de especialización de posgrado destinado a egresadas de Escuelas Normales e Institutos Superiores del Profesorado Elemental, para el Nivel Pre-escolar/Jardín de Infantes.

Como coordinador del mencionado curso estaba el profesor Félix Cosme Schmid, el cual solicita al Decano se designe personal especializado en el Nivel Pre-Elemental para que colabore en la organización, quedando afectadas a dicha tarea la Inspectora Técnica de Enseñanza Pre-Elemental de la provincia, Prof. Berta Bocco de Varisco, la Directora del Jardín de Infantes provincial $N^{\circ} 5$, Prof. María Mercedes Duprat de Martina y la Profesora Nélida Sosio de Iturrióz (11), quien es designada como Secretaria Técnica de la Comisión encargada de la organización de dicho Profesorado (12).

Entre otros datos que se fueron presentando en el apartado anterior (matrícula escolar, cantidad de establecimientos, etc.), vale resaltar que el informe elaborado por la Comisión mencionada aportó a la comprensión de la situación en que se desarrollaba la educación pre-escolar, en las siguientes dimensiones fundamentales:

- Necesidad de instalar la educación inicial en la región a partir de la definición de políticas estatales que aseguren y sostengan un adecuado desarrollo y expansión.

- ' 'Imperiosa' necesidad de preparación del personal docente.

- Se informa vacíos existentes en el desarrollo de este nivel, por ejemplo, carencias en la supervisión de los establecimientos de enseñanza preescolar, dado que un único inspector debía atender 22 establecimientos con 58 secciones.

- Insuficiencia en los materiales didácticos también para garantizar un trabajo adecuado en los establecimientos.

- Necesidad de mejoramiento de los edificios escolares y creación de nuevos establecimientos educativos para el nivel.

Como consecuencia de este informe, en diciembre de 1972 el primer curso dictado pasa a denominarse Profesorado en Educación Pre-Elemental, con carácter de carrera a término (por Resolución N 4933/72). Por la misma Resolución, se crea el Departamento de Educación Pre-Elemental por el lapso que dure dicha carrera, designándose Director del Departamento al Profesor Félix Schmid, a la Profesora Mercedes Dulce Corsi como Vicedirectora y a la Profesora Nélida Sosio de Iturrióz en carácter de Secretaria Técnica.

Otra nueva Resolución, la N 58/73-C.S. crea la Carrera del Profesorado en Educación Pre-Elemental (13) - a término, dependiente de la Facultad de Humanidades de la UNNE (14).

En los inicios y en el desarrollo de la carrera la presencia de Margarita Ravioli fue constante (15), y Nélida mantuvo contacto frecuente con ella: 
Revista de la Escuela de Ciencias de la Educación, año 12, nRo.11, vol. 1, enero a Junio de 2016. Páginas 65-78. ISSN 1851-6297. ISSN EN LINEA 2362-3349. HISTORIA DE LA FORMACIÓN DE DOCENTES PARA EL NIVEL INICIAL EN EL CHACO: la conformación de la carrera de Profesorado de Educación Pre-elemental en la Universidad Nacional del Nordeste (1973-1982). Alcides David Musín - Ileana Ramirez - Victoria Soledad Almiron

(...) hice todo un trabajo y se lo lleve a Margarita para que lo viera y le gusto, era un trabajo diagnóstico sobre la necesidad que había entonces, ese trabajo fue al Consejo de Rectores de Universidades Nacionales de Buenos Aires y les pareció que realmente había una necesidad y entonces se creó el profesorado.

(...) Los viajes de estudio a Buenos Aires duraban una semana. Íbamos a recorrer los jardines, al cine, al teatro, a bailar. Lo primero, íbamos al Eccleston. Con la primera camada pude darme el lujo de llevarlas al Mitre, en septiembre del '76 fuimos y nos recibió Margarita y me dí el gusto de llevar a mis alumnas a que conozcan (Ibídem).

Para el ingreso a la carrera se efectuó un llamado a inscripción que alcanzó un total de 148 postulantes, de las cuales quedaron seleccionadas 56. Al igual que en el Instituto "Sara Ch. de Eccleston", para el ingreso se les tomaba examen.

La estructura organizativa, pedagógica-curricular del profesorado tenía por propósitos formar profesoras para la región de influencia de la UNNE, lo que suponía:

- Prepararlos filosóficamente, para elaborar los fines y objetivos de la educación pre-elemental, en relación con los de la educación del país.

- Prepararlos psicológicamente, para conocer las características del niño de 0 a 7 años.

- Tomar conciencia de la realidad socio-económica de la Nación y de la Región, con el fin de que se integren al ámbito cultural, nacional y regional.

- Sociológicamente, orientarlo en la interpretación de las necesidades educacionales de la comunidad donde funcionen los establecimientos pre-elementales: satisfacer las necesidades de desarrollo educativo del nivel, en relación con los otros niveles educativos, en el orden regional y en estrecha vinculación con el Nacional (Informe, 1972, p.3)

El plan de estudios comprendía 26 materias, con una carga horaria total de 2960 horas reloj de clases teórico-prácticas. Las materias estaban distribuidas en 5 cuatrimestres.

Si bien no es objetivo de esta trabajo detenernos en un análisis del plan de estudio, podemos mencionar en términos generales que el mismo contenía: el estudio de los precursores universales de la educación infantil (Fröebel, Agazzi, Montessori, Decroly); una fuerte impronta psicológica y didactista; la práctica como eje estructurante, que aparece desde el primer cuatrimestre; literatura infantil; la formación cultural (por ejemplo actividades culturales y educativas como viajes a Buenos Aires donde visitaban teatros, jardines, etc.). De lo mencionado podemos evidenciar una clara impronta del Instituto Sara Ch. de Eccleston en la propuesta curricular formulada. 
Revista de la Escuela de Ciencias de la Educación, año12, nRo.11, vol. 1, enero a Junio de 2016. Páginas 65-78. ISSN 1851-6297. ISSN EN LINEA 2362-3349. HISTORIA DE LA FORMACIÓN DE DOCENTES PARA EL NIVEL INICIAL EN EL CHACO: la conformación de la carrera de Profesorado de Educación Pre-elemental en la Universidad Nacional del Nordeste (1973-1982). Alcides David Musín - Ileana Ramirez - Victoria Soledad Almiron

\section{Conclusiones}

Retomando las preguntas planteadas al inicio, este artículo evidencia la complejidad inherente al campo educativo, y específicamente, en la pretensión histórica de instalar y consolidar el estatuto pedagógico del nivel. En esta oportunidad nos focalizamos en la formación docente para la educación preescolar, como un campo de tensiones entre perspectivas y enfoques de corte pedagógico-didáctico, psicológico y filosófico.

Desde el recorrido por la formación de Nélida en el Instituto Sara Ch. de Eccleston pudimos reflexionar acerca de cómo las huellas de su trayectoria imprimieron determinadas idiosincrasias a la estructuración de la carrera en la UNNE, y que estuvieron expresadas en la selección de teorías pedagógicodidácticas, en las concepciones que se sostenían sobre la infancia, en la función otorgada al jardín de infantes, entre otras.

Asimismo, las fuentes consultadas nos permiten, por una parte, problematizar y discutir con el imaginario que representaba como "un desierto" al desarrollo de la educación infantil en el Chaco en los '70. En este periodo se registró un movimiento de ampliación, en cuanto a la cantidad de establecimientos creados, y de expansión territorial de la educación pre-escolar en la provincia. Esto instaló una demanda real fundamentada para la propuesta pedagógica de formación específica en el nivel y que se plasmó en una formación integral y amplia (filosófica, cultural, psicológica, biológica) por sobre una capacitación acotada como se venía desarrollando en la provincia.

\section{Notas Bibliográficas}

(1) El artículo se enmarca en el proyecto de investigación en curso "La formación de docentes en educación infantil: Entre el neoliberalismo y la construcción de ciudadanía. (PI H008-2013. CyT-UNNE. Res N 678/13 CS.) Director: José I. Rivas Flores.

Tomamos el periodo correspondiente a los orígenes de la Carrera como propuesta académica a término hasta el año en que se constituye en carácter de carrera permanente.

(2) A partir de modificaciones curriculares posteriormente esta propuesta inicial fue sufriendo transformaciones dando lugar a lo que actualmente es el Profesorado de Educación Inicial.

(3) Por Resolución N 4934/72-C.A. se crea el Departamento de Educación Pre Elemental en el ámbito de la Facultad de Humanidades, y por el lapso que dure el dictado de dicha carrera a término. Se designa entonces al profesor Félix Cosme Schmid como Director del citado Departamento, a la Profesora Mercedes Dulce Corsi como Vicedirectora y a la Profesora Nélida Sosio de Iturrióz como Secretaria Técnica.

(4) Profesorado en Educación Pre-Elemental. Bases Generales para la Estructuración del Profesorado en Educación Pre-Elemental (1972). Facultad de Humanidades, Universidad Nacional del Nordeste.

(5) Respecto a la formación específica de docentes para el nivel, la organización formulaba: “(...) La O.M.E.P insiste en que antes de crear Instituciones para la atención del preescolar, es indispensable disponer del personal especializado; para lo cual, y con el fin de evitar imprudentes improvisaciones que podrían comprometer el futuro de los niños, 
Revista de la Escuela de Ciencias de la Educación, año 12, nRo.11, vol. 1, enero a Junio de 2016. Páginas 65-78. ISSN 1851-6297. ISSN EN LINEA 2362-3349. HISTORIA DE LA FORMACIÓN DE DOCENTES PARA EL NIVEL INICIAL EN EL CHACO: la conformación de la carrera de Profesorado de Educación Pre-elemental en la Universidad Nacional del Nordeste (1973-1982). Alcides David Musín - Ileana Ramirez - Victoria Soledad Almiron

será preciso crear los Institutos Superiores que provean profesionales y educadoras de alta competencia técnica, comprensión de la naturaleza humana y vocación de servicio." Discurso inaugural del Primer Congreso Argentino de Educación Preescolar. Publicado en el Boletín № 2 (1969) del Comité Argentino de la O.M.E.P.

(6) La organización y dirección del Instituto se encomendó a la Profesora Margarita Ravioli, de quien hicimos referencia en el cuerpo del trabajo. A su cargo estuvo la preparación de los planes y programas del curso y el profesorado que dirigió por espacio de casi cuarenta años.

(7) La cual se vio plasmada en la creación de 636 secciones de jardines de infantes anexas a escuelas comunes de la Capital, provincias y Territorios Nacionales.

(8) Los datos presentados aquí fueron extraídos del Informe "Profesorado en Educación Pre-Elemental. Bases Generales para la Estructuración del Profesorado en Educación Pre-Elemental" (Agosto de 1972). Facultad de Humanidades, Universidad Nacional del Nordeste y del "Informe y análisis de la situación del nivel pre-escolar en la provincia del Chaco". Asociación de Maestras Jardineras Chaco (Junio de 1972).

(9) Por Decreto $\mathrm{N}^{\circ} 1945 / 54$

(10) A modo de ilustrar comparativamente, para el año 1952 había un total de 483 escuelas primarias. Entre 1954 y 1978 se crearon un promedio de 255 establecimientos primarios en toda la provincia (De Pompert de Valenzuela, 2006).

(11) Por Resolución N 653/72 del Consejo General de Educación de la provincia del Chaco.

(12) Esta Comisión fue la encargada de elaborar el informe de diagnóstico al que hicimos referencia en nuestro análisis.

(13) El Profesorado en Educación Pre elemental, a término, con una duración de dos años y un cuatrimestre, tendrá como "objetivo fundamental formar al profesor en educación pre-elemental en forma sólida y coherente, con una fundamentación y base científica a la vez que práctica, para que tome conciencia de la realidad socio económica de la Nación y de la región, con el fin de que se integre al ámbito cultural, nacional y regional; orientándolo en la interpretación de las necesidades educacionales de la comunidad.

Se trata de esta forma de preparar personal altamente capacitado para poder así satisfacer las necesidades del desarrollo del nivel Pre elemental en el orden regional. Al ser a término (el profesorado antes mencionado), sólo se recibirán un grupo de alrededor de 60 profesionales, siendo necesario cubrir las necesidades ocupacionales de la zona de influencia de la Universidad Nacional del Nordeste, que abarca las provincias de Chaco, Misiones, Formosa y Corrientes. Por tal motivo, la Facultad de Humanidades está estudiando la posibilidad de crear en forma estable la Carrera del Profesorado en Educación Pre elemental en la UNNE".

(14) El 28 de marzo de 1973 se firmó un convenio (Decreto $N^{\circ} 702 / 73$ ) entre el Ministerio de Gobierno, Justicia y Educación de la provincia del Chaco y la Facultad de Humanidades de la UNNE para intervenir mancomunada y planificadamente en el funcionamiento a término del Profesorado de referencia. El 18 de mayo del citado año, el Consejo de Rectores de las Universidades Nacionales resuelve "Aprobar la creación del Profesorado en Educación Pre-Elemental -a término- en el ámbito de la Universidad Nacional del Nordeste".

(15) Margarita fallece en Buenos Aires el $1^{\circ}$ de marzo de 1977. En abril de 1979 se recibió, por parte de los familiares de la profesora Marina Margarita Ravioli, la donación de su biblioteca personal al Departamento de Educación Pre elemental de la Facultad de Humanidades de la UNNE. Este acervo bibliográfico consta de 234 fichas bibliográficas de Autor, 239 fichas bibliográficas de Título y 320 fichas bibliográficas de Materia. El trabajo de catalogación, fichado y archivo estuvo a cargo de un grupo de alumnas practicantes 
Revista de la Escuela de Ciencias de la Educación, año12, nRo.11, vol. 1, enero a Junio de 2016. Páginas 65-78. ISSN 1851-6297. ISSN EN LINEA 2362-3349. HISTORIA DE LA FORMACIÓN DE DOCENTES PARA EL NIVEL INICIAL EN EL CHACO: la conformación de la carrera de Profesorado de Educación Pre-elemental en la Universidad Nacional del Nordeste (1973-1982). Alcides David Musín - Ileana Ramirez - Victoria Soledad Almiron

de la carrera de Ciencias de la Información de dicha Facultad, bajo la supervisión del equipo docente de la cátedra Historia de la Educación Infantil del Departamento de Educación Inicial.

\section{Referencias bibliográficas}

- Carli, S. (2002). Niñez, pedagogía y política. Transformaciones de los discursos acerca de la infancia en la historia de la educación argentina entre 1880 y 1955. Buenos Aires: Miño y Dávila editores.

- De Pompert de Valenzuela, M. C. (2006). Historia de la educación primaria en el Chaco. 1872-1978. Resistencia: Librería de la Paz.

- $\quad$ Diker, G. \& Terigi, F. (2003). La formación de maestros profesores: hoja de ruta. Buenos Aires: Paidós.

- Ponce, R. (2006). Los debates de la educación inicial en la Argentina. Persistencias, transformaciones y resignificaciones a lo largo de la historia. En Malajovich, A. (comp.) Experiencias y reflexiones sobre la educación inicial. Una mirada latinoamericana (19101). Buenos Aires: Siglo XXI editores.

- Väg, O. (1991). La investigación en historia de la educación preescolar: algunos asuntos a debatir. Historia de la Educación, 10,15-20.

- Vasta, L., y Gispert F. (2009). Recuperando la trama fundacional: orígenes del Profesorado de Educación Inicial Sara C. de Eccleston y del Jardín de Infancia Mitre. e-Eccleston. Temas de Educación Infantil, №12, 8-19.

\section{Fuentes primarias}

- Entrevista a Nélida Sosio de Iturrioz. Resistencia, 2013.

- Informe y análisis de la situación del nivel pre-escolar en la provincia del Chaco. Asociación de Maestras Jardineras Chaco (junio de 1972).

- Profesorado en Educación Pre-Elemental. Bases Generales para la Estructuración del Profesorado en Educación Pre-Elemental (agosto de 1972). Facultad de Humanidades, Universidad Nacional del Nordeste. 